\title{
Complex Scalar Field Dark Matter and its Imprint on Primordial Gravitational Waves from Inflation
}

\section{Bohua Li*}

The University of Texas at Austin

E-mail: bohualieastro.as.utexas.edu

\section{Paul R. Shapiro}

The University of Texas at Austin

E-mail: shapirodastro.as.utexas.edu

\section{Tanja Rindler-Daller}

University of Michigan/University of Vienna

E-mail: daller@umich.edu

Recently there has been an increasing interest in an alternative dark matter candidate, ultralight bosonic dark matter $\left(m \gtrsim 10^{-22} \mathrm{eV}\right)$. Among its various types of models, we propose that dark matter is comprised of complex scalar bosons, for which the charge (particle number) per unit comoving volume is conserved, fixed by matching the observed dark matter density at present. The enormous charge density guarantees that ultralight bosons form a Bose-Einstein condensate (BEC) immediately after their particle production during a standard $(w=0)$ reheating process. When the homogeneous background universe evolves in the presence of this form of scalar field dark matter (SFDM), the equation of state of SFDM is relativistic at early times, evolving from stiff $(\bar{p}=\bar{\rho})$ to radiationlike $(\bar{p}=\bar{\rho} / 3)$, before it becomes nonrelativistic and CDM-like at late times $(\bar{p}=0)$. Therefore, as an extra relativistic species, SFDM contributes to the Hubble expansion rate in the early universe. In fact, SFDM even dominates the total energy density of the universe when it is in the stiff phase. The timing of transitions between these phases is determined by SFDM model parameters, particle mass $m$ and self-interaction coupling strength $\lambda$. They can be constrained by cosmological observables, particularly $N_{\text {eff }}$, the effective number of neutrino species during BBN, and $z_{\mathrm{eq}}$, the redshift of matter-radiation equality. In addition, SFDM affects the homogeneous evolution of the universe via the amplification of primordial gravitational waves (GWs) by the stiff phase. Primordial tensor modes that reenter the horizon during the stiff phase contribute a higher energy density to the stochastic GW background than they would have in the standard model with only a radiation-dominated phase. This increases the expansion rate of the radiation-dominated phase with consequences for the observables above as well and makes possible the detection of these high-frequency GWs today by laser interferometer experiments. We find significant constraints on SFDM particle parameters, $m$ and $\lambda /\left(m c^{2}\right)^{2}$, for currently allowed values of the tensor-to-scalar ratio $r$ and reheat temperature $T_{\mathrm{re}}$.

BASH 2015

18 - 20 October, 2015

The University of Texas at Austin, USA

\footnotetext{
${ }^{*}$ Speaker.
} 
Complex SFDM in BEC can be described by a classical scalar field $\psi$. In standard units, the potential has a $m|\psi|^{2}$ term, in which $m$ is the mass, and a repulsive self-interaction term $\lambda|\psi|^{4}$, in which $\lambda$ is the self-interaction coupling strength. The complex scalar field satisfies charge conservation due to its $U(1)$ symmetry, and the charge density is determined by the present-day dark matter abundance. We solve for the evolution of a homogeneous $\Lambda$ SFDM universe for which dark matter is in the form of SFDM and cosmological parameters are set by present values measured by Planck [1], now taking self-consistent account of the GW background from inflation as well [2]. The existence of the stiff phase of SFDM is shown in Fig. 1 (upper-left).

Standard inflation seeds tensor fluctuations of which the initial power spectrum depends on tensor-to-scalar ratio $r$. As a main source to the $B$-mode polarization of $\mathrm{CMB}$, primordial tensor fluctuations are now constrained by $\mathrm{CMB}$ experiments through the $B B$ power spectrum. On the other hand, they add to the mean energy density of the universe as a stochastic GW background once they enter the horizon. If they enter the horizon during the stiff phase $(w=1)$, their energy density is boosted relative to what it would be if they enter during the radiation-dominated phase, reflected in the present-day GW energy spectrum $\mathrm{d} \Omega_{\mathrm{GW}} / \mathrm{d} \ln k$ as a change in the spectral index. See Fig 1 (bottom). This excess radiation is subject to the constraint from $N_{\text {eff }}$ during BBN and $z_{\text {eq }}$. We require that $N_{\text {eff }}$ in $\Lambda$ SFDM be within $1 \sigma$ limit of the measured value during BBN, from $a_{\mathrm{n} / \mathrm{p}}$ at neutron/proton ratio freeze-out to $a_{\text {nuc }}$ at light element production. See Fig 1 (upper-right).
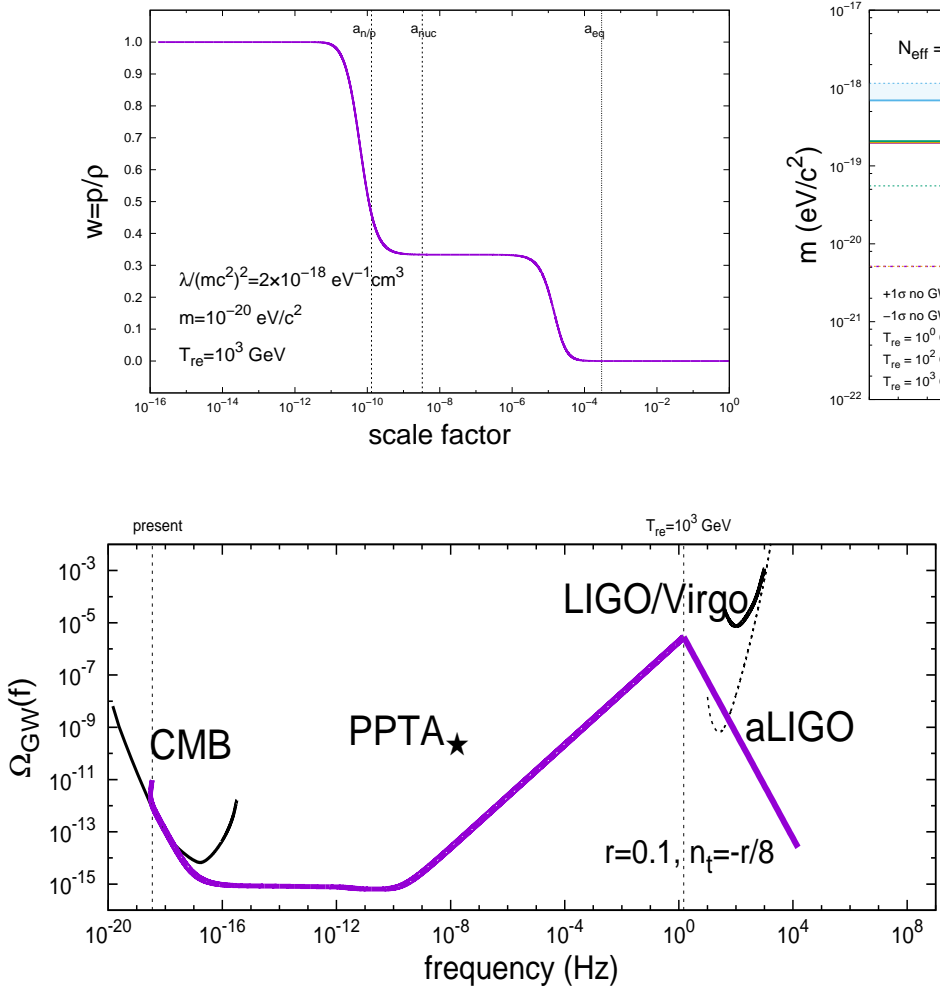

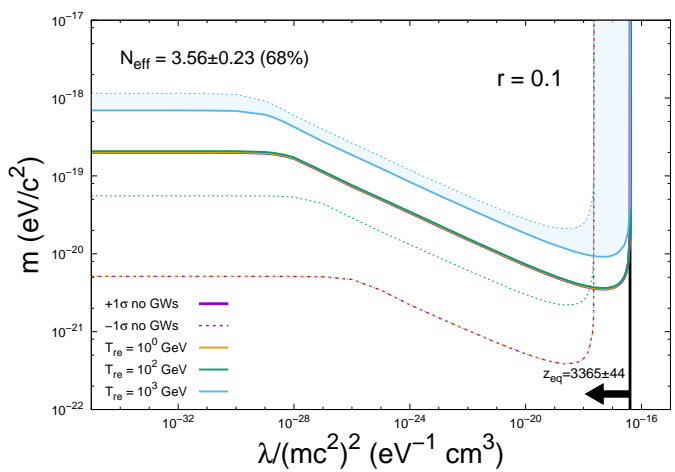

Figure 1: (upper-left) Evolution of SFDM equation of state for a fiducial choice of parameters. (upper-right) SFDM particle parameter space. The blue shaded region is allowed if $T_{\mathrm{re}}=$ $10^{3} \mathrm{GeV}$. (bottom) GW spectrum today for the fiducial $\Lambda$ SFDM universe. Black curves and the star (current best limit from the Parkes Pulsar Timing Array) are data from various experiments, provided by Lasky et. al. [3].

\section{References}

[1] Li, B., Rindler-Daller, T., \& Shapiro, P. R. 2014, Phys. Rev. D 89, 083536

[2] Li, B., Shapiro, P. R., \& Rindler-Daller, T., in preparation

[3] Lasky, P. D., Mingarelli, C. M. F., Smith, T. L., et al. 2015, arXiv:1511.05994 